\title{
Gastrointestinal Disorders in Parkinson's Disease: Prevalence and Health Outcomes in a US Claims Database
}

\author{
Lydia Makaroff ${ }^{\mathrm{a}}$, Angus Gunn ${ }^{\mathrm{a}}$, Christophe Gervasoni ${ }^{\mathrm{b}}$ and Florent Richy ${ }^{\mathrm{a}, \mathrm{c}, *}$ \\ ${ }^{a}$ UCB Pharma S.A., Allée de la Recherche, Brussels, Belgium \\ ${ }^{\mathrm{b}}$ Keyrus Biopharma, Chaussée de Louvain, Lasne, Belgium \\ ${ }^{\mathrm{c}}$ Public Health, Epidemiology and Health Economics, University of Liège, Liège, Belgium
}

\begin{abstract}
The majority of patients with Parkinson's Disease (PD) will eventually develop gastrointestinal disorders (GIDs) such as dysphagia, constipation and gastroesophageal reflux. The objectives of this study were to examine the incidence of GIDs in PD patients in a US population, and to examine subsequent PD-related outcomes in patients with GIDs. In a US administrative health claims database, GID incidence increased over time to reach $65 \%$ at four years after PD diagnosis. To further investigate this relationship, a subset of patients was analysed in greater detail. Continuously treated PD patients with and without GIDs were matched by age, gender, comorbidities, treatment regime, US region and plan type. Their emerging health outcomes were followed up for two years. Outcomes included neuropsychiatric, motor and urogenital disturbances, as well as healthcare utilization and costs. Patients with GIDs had higher rates of psychosexual dysfunction, anxiety, depression, ataxia, pain, movement disorders, urinary incontinence and falls. Emergency room admissions, the number of drugs for pain, sleep and depression, PD-related healthcare costs and non PD-related healthcare costs also increased during the observation period in GID patients. This study indicated that GIDs may be associated with deleterious effects on some PD-related outcomes.
\end{abstract}

Keywords: Parkinson disease, gastrointestinal diseases, health care economics and organizations, outcome assessment (health care)

\section{INTRODUCTION}

Parkinson's disease (PD) is a progressive neurological condition first described in 1817 [1]. This disease is linked to a deficiency of the dopamine neurotransmitter, required to transmit signals that regulate motor functions such as walking and balance. The key symptoms are tremor, rigidity, bradykinesia and postural instability [2]. PD has an estimated age-adjusted prevalence of 3.63 per 1000 men and 3.24 per 1000 women, and the risk of disease increases with age [3].

${ }^{*}$ Correspondence to: Dr Florent Richy, Head of Epidemiology, UCB Pharma S.A., Allée de la Recherche, 60, 1070 Brussels, Belgium. Tel.: +32 2559 9327; Fax: +32 2559 9370; E-mail: Florent.Richy@ucb.com.
Many patients with PD also suffer from dysfunctions of mood, behaviour, sleep, sensation and autonomic function. These non-motor symptoms have been observed to increase with age, although symptoms such as olfactory problems, constipation and depression can occur quite early in the disease [4]. Gastrointestinal disorders (GIDs) are the most commonly observed non-motor symptom of PD, affecting nearly all aspects of the gastrointestinal system. Some of the more common symptoms include dysphagia, gastroparesis and bowel dysfunction [5]. Disorders in gastrointestinal motility are often found even in the very early stages of PD, though patients may be unlikely to report mild symptoms unless specifically questioned about these issues [6]. Gastrointestinal symptoms tend to worsen as the disease progresses [7]. 
While there is currently no cure for PD, functional impairment is usually treated with levodopa and/or dopamine agonists (DA). The standard of care for most patients is to first place the patient on monotherapy and then on polytherapy if the disease progresses in severity [2]. Early treatment of patients with DA has been associated with a reduced risk of motor complications [8].

Delayed gastric emptying and, thus, delayed drug influx to the intestine may result in inconsistent reactions to orally-delivered medication. Furthermore, retention of levodopa in the stomach lengthens its exposure to dopa-decarboxylase, which can make it less available for absorption in the duodenum [9]. These variations in peripheral pharmacodynamics may account for some of the motor and non-motor fluctuations observed in individuals with PD [10].

Most of these non-motor symptoms have been studied within the context of clinical trials. While these studies are essential to prove the safety and efficacy of medications, they may not be the best environment for observing prolonged longitudinal changes in a large body of patients. Administrative claims databases provide an opportunity to extend the results of clinical trials by examining the long-term progression of disease in an observational setting [11]. This study was designed to examine the frequency of GIDs in patients in the general US population with PD, and to determine the association between GID diagnosis and subsequent health-care outcomes.

\section{METHODS}

\section{Database}

Data were obtained using the PharMetrics PatientCentric Database (IMS Health, Connecticut, USA) that comprises of de-identified information from enrolment files as well as facility, professional service and outpatient pharmacy claims from private healthcare benefit plans covering over 55 million patients enrolled in more than 90 health plans across the United States. All claims for a given patient are linked using a unique encrypted identifier. The dataset is compliant with the Health Insurance Portability and Accountability Act (HIPAA).

\section{Study design - Kaplan-Meier curve analysis}

In order to determine the rate of GID diagnosis in PD patients, Kaplan-Meier estimates were constructed. Patients were divided into three groups based on age: younger than 65 years, between 65 and 75 years, and older than 75 years at the time of PD diagnosis. First, all patients with continuous enrolment for at least 12 months before PD diagnosis were analysed to determine the proportion of patients who had a GID diagnosis within 12 months prior to their PD diagnosis. To assess the proportion of GID diagnoses after PD diagnosis, only patients with greater than $80 \%$ medicine compliance ratio (see below) were included in the analysis. The inclusion criteria are listed in Table 1 .

\section{Population}

The study population consisted of patients who had at least one claim between January 2000 and December 2008 that was associated with a diagnosis for PD using the International Classification of Diseases, Ninth Revision (ICD-9 332.xx). The index date was the date of the first diagnosis for PD.

Table 1

Inclusion criteria used for Kaplan-Meier analysis

\begin{tabular}{lr}
\hline Criterion & Number of patients \\
\hline GID incidence before PD diagnosis & 75,643 \\
All patients in the datacut with at least one diagnosis with PD (index date: date of diagnosis) & 32,877 \\
Patients have continuous enrolment during the 12 month (365 day) pre-index period & 8,485 \\
Patients are <65 years old at index date & 8,731 \\
Patients are 65-75 years old at index date & 15,661 \\
Patients are $>75$ years old at index date & 23,964 \\
GID incidence after PD diagnosis & \\
All patients in the datacut with at least one diagnosis with PD (index date: date of diagnosis) & 3,116 \\
AND patients do not have a GID during the 12 month (365 day) pre-index period & 975 \\
Patients have $>80 \%$ MPR & 992 \\
Patients are <65 years old at index date & 1,149 \\
Patients are 65-75 years old at index date & \\
Patients are $>75$ years old at index date &
\end{tabular}




\section{Measurements}

Claims during the pre- and post-index period were scanned to identify PD cases that had one or more diagnosis for a GID of interest.

\section{Medication possession ratio}

Pharmacy claims during the post-index period were scanned to calculate for each patient the number of prescriptions for levodopa and DA. Pharmacy records have shown to be a valid measure of the exposure of patients to their medication [12]. Prescriptions reported as filled were assumed to be prescriptions taken by the patient. These data were used to calculate the medication possession ratio (MPR), and thus the adherence of the patients to their regime. The MPR was calculated as the sum of the total days supplied on all levodopa or DA prescriptions, divided by the length of the period from index date to event date. To be included in the postPD diagnosis GID incidence analysis, patients were required to have an MPR greater than or equal to $80 \%$ for levodopa and/or $80 \%$ for DA.

\section{Study design - Case-control propensity matched analysis}

The study hypothesis was that patients with GIDs may have worse PD-related outcomes. A literature review and expert clinician consultation was conducted to determine appropriate outcome measures [4]. All comorbidities generated by this review process were subdivided into the categories of neuropsychiatric, motor, urogenital, cardiovascular, and falls and frac- tures, using corresponding ICD-9 codes. Comparisons were made between patients who had a diagnosis for a GID in the pre-index period and patients who did not have a diagnosis for a GID in the pre-index period (see Fig. 1). The inclusion criteria are listed in Table 2.

\section{Population}

The study population consisted of patients with PD who had at least two claims that were associated with a diagnosis for PD. The study captured patients who had prescriptions for levodopa or DA between September 2005 and September 2006. The index date was the date of the first levodopa or DA prescription after the first diagnosis for PD. Levodopa and DA were chosen as the target medications as these are the treatments that are most commonly recommended to US physicians [2]. Patients must have maintained continuous enrolment during the entire six month pre-index and two year post-index period.

\section{Measurements}

Age, sex, US region, and healthcare benefit plan type at the index date were obtained from enrolment files. Claims during the pre-index period were scanned to identify PD cases who had one or more diagnosis for a GID of interest (listed in Table 3), or control PD patients who did not have any diagnosis for the GIDs of interest during the pre-index period.

\section{Medication possession ratio}

The MPR was calculated as the sum of the total days supplied on all levodopa or DA prescriptions, divided

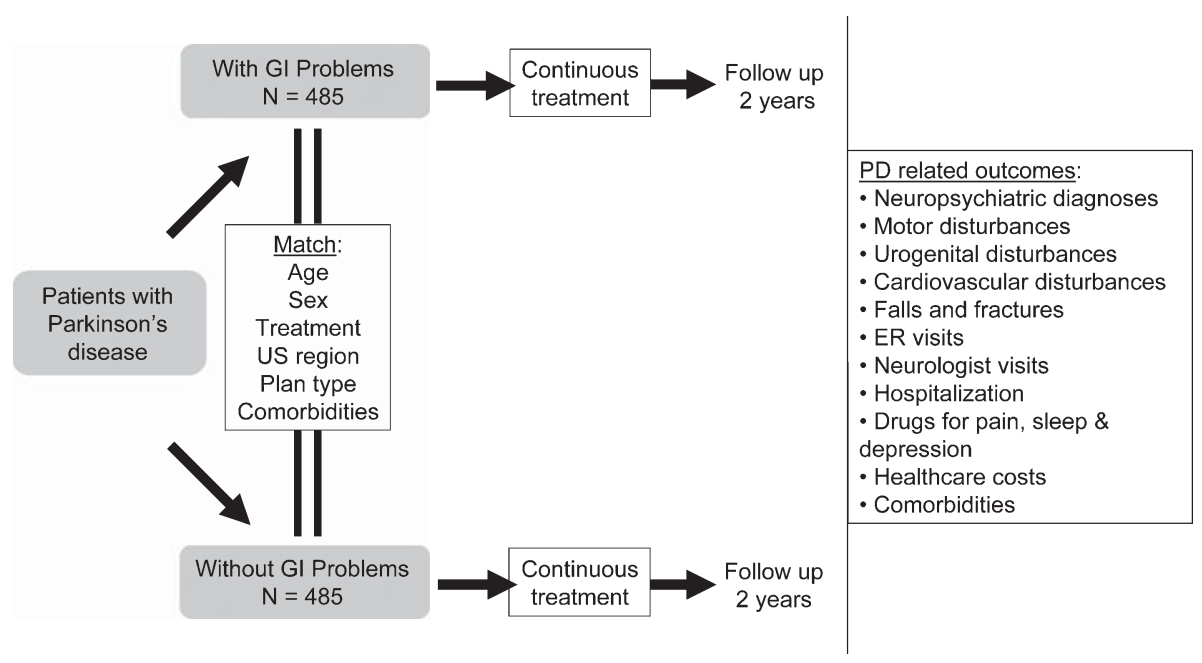

Fig. 1. Experimental design of matched patient analysis of compliant PD patients with GIDs matched to compliant PD patients without GIDs. 
Table 2

Inclusion criteria used for the case-control health-outcomes analysis

\begin{tabular}{lc}
\hline Criterion & Number of patients \\
\hline Patients have a prescription for Levodopa and/or DA between 1 September 2005 and 1 September & 15,579 \\
2006 (index date: date of first prescription) & 7,851 \\
Patients have continuous enrolment during 6 month (180 day) pre-index period AND patients have & 5,529 \\
$\quad$ continuous enrolment during 24 month (730 day) post-index period & 3,177 \\
Patients have at least two diagnoses for PD during 6 month (180 day) during pre-index period & $486(485$ matched*) \\
Patients have an MPR $\geq 0.8$ for a Levodopa and/or an MPR $\geq 0.8$ for DA & 2,691 (485 matched*) \\
Cases: patients have $\geq 1$ diagnosis for GI disorders during the pre-index period & Controls: patients without a GI disorder during the pre-index period \\
*Each case is matched with only 1 control (1: 1 match) on propensity score using an $8>1$ digit match [14]. Incomplete matching \\
for 1 case out of 486 was lost due to a failure to match on a specified number of digits $(8>1)$, and thus this patient was excluded \\
from the analysis.
\end{tabular}

Table 3

ICD-9 diagnosis codes used to identify patients with gastrointestinal disorders

\begin{tabular}{|c|c|c|}
\hline ICD-9 code & Diagnosis & Description \\
\hline $787.2 \mathrm{x}$ & Dysphagia & Dysphagia \\
\hline 530.xx & Esophagal disease & Diseases of the esophagus \\
\hline 531.xx & Gastric ulcer & Gastric ulcer \\
\hline 532.xx & Duodenal ulcer & Duodenal ulcer \\
\hline 533.xx & Peptic ulcer & Peptic ulcer, site unspecified \\
\hline 534.xx & Gastrojejunal ulcer & Gastrojejunal ulcer \\
\hline 535.xx & Gastritis/duodenitis & Gastritis and duodenitis \\
\hline 536.xx & Disorders of stomach function & Disorders of function of stomach \\
\hline 537.xx & Stomach/duodenum disorders & Other disorders of stomach and duodenum \\
\hline 538.xx & Gastrointestinal mucositis & Gastrointestinal mucositis (ulcerative) \\
\hline 560.xx & Intestinal obstruction & Intestinal obstruction without mention of hernia \\
\hline 564.xx & Digestive disorders & Functional digestive disorders, not elsewhere classified \\
\hline 568.xx & Peritoneum disorders & Other disorders of peritoneum \\
\hline 569.xx & Intestinal disorders & Other disorders of intestine \\
\hline
\end{tabular}

by the length of the post-index period ( 24 months). To be included in the study, patients were required to have an MPR greater than or equal to $80 \%$ for levodopa and/or $80 \%$ for DA. Patients with an MPR of less than $80 \%$ were excluded from the study. This subset of patients was selected to ensure that the results were not influenced by worse outcomes due to non-compliance.

\section{Outcomes}

Claims were assumed to be PD-related if they included a diagnosis of PD. The number of prescriptions for drugs used to treat pain, depression and sleep disorders were also monitored over the postindex period. Based on reported diagnoses, Deyo's version of the Charlson comorbidity index (CCI) [13] was used to produce a weighted index of 17 chronic medical conditions for each patient. For the determination of healthcare costs, the PharMetrics variable "ALLOWED" was examined, which reflects the paid amount plus any member liability, as it is the cost variable that most closely reflects the burden of illness.

\section{Statistical analysis}

Data were analysed with the SAS statistical package (version 9.1; SAS Institute Inc., North Carolina, USA). Descriptive analyses (frequencies and percentages) of baseline characteristics were performed on the patients in the GIDs group. Patients for the "No GIDs" group were selected using a propensity-matching system that took into account the baseline characteristics of age, sex, treatment regime, US region, plan type and pre-index CCI [14]. Hypothesis testing was conducted using McNemar's tests for categorical variables and Wilcoxon signed rank sum tests for continuous variables. The nominal significance threshold was not adjusted to take into account multiple testing. All $p$-values can only be interpreted in an explorative manner.

\section{RESULTS}

Survival curves from a retrospective analysis of a US administrative health claims database showed an increased incidence over time of GIDs in patients diagnosed with PD. This analysis found that $29 \%$ of 
patients who were diagnosed with PD had been diagnosed with GIDs in the previous year. By four years post PD diagnosis the proportion of patients who had been diagnosed with GIDs was $50 \%$ for patients under $65,68 \%$ for patients between 65 and 75 , and $72 \%$ for patients over 75 (Fig. 2).

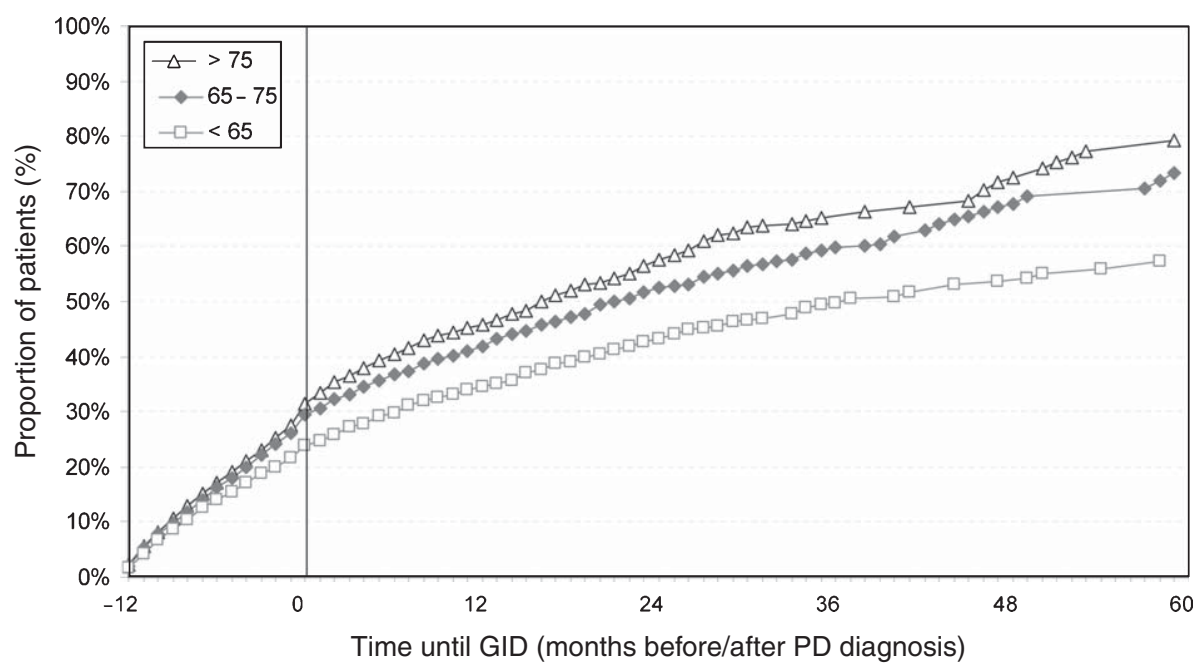

Fig. 2. Proportion of all compliant PD patients (\%) by time (months before or after PD diagnosis) until their first diagnosis of a GID. Results are shown for patients aged $<65$ years, $65-75$ years, and $>75$ years.

Table 4

Characteristics of the compliant PD patients with GIDs and the matched control population of compliant PD patients without GIDs

\begin{tabular}{|c|c|c|c|}
\hline & $\begin{array}{c}\text { No GI disorder } \\
N(\%)\end{array}$ & $\begin{array}{c}\text { GI disorder } \\
\qquad N(\%)\end{array}$ & $\begin{array}{c}\text { Total } \\
N(\%)\end{array}$ \\
\hline \multicolumn{4}{|l|}{ Age group (years) } \\
\hline $0-34$ & $0(0.0)$ & $0(0.0)$ & $0(0.0)$ \\
\hline $35-44$ & $1(0.2)$ & $0(0.0)$ & $1(0.1)$ \\
\hline $45-54$ & $11(2.3)$ & $18(3.7)$ & $29(3.0)$ \\
\hline $55-64$ & $83(17.1)$ & $94(19.4)$ & $177(18.2)$ \\
\hline $65-74$ & 145 (29.9) & $142(29.3)$ & $287(29.6)$ \\
\hline $75-84$ & $180(37.1)$ & $170(35.1)$ & $350(36.1)$ \\
\hline $85+$ & $65(13.4)$ & $61(12.6)$ & $126(13.0)$ \\
\hline \multicolumn{4}{|l|}{ Gender } \\
\hline Men & $270(55.7)$ & $257(53.0)$ & $527(54.3)$ \\
\hline Women & $215(44.3)$ & $228(47.0)$ & $443(45.7)$ \\
\hline \multicolumn{4}{|l|}{ Payor type } \\
\hline Commercial & $382(78.8)$ & $371(76.5)$ & $753(77.6)$ \\
\hline Medicaid & $3(0.6)$ & $5(1.0)$ & $8(0.8)$ \\
\hline Medicare risk & $54(11.1)$ & $61(12.6)$ & 115 (11.9) \\
\hline Self-insured & $5(1.0)$ & $6(1.2)$ & $11(1.1)$ \\
\hline Medicare gap & $41(8.5)$ & $42(8.7)$ & $83(8.6)$ \\
\hline \multicolumn{4}{|l|}{ Geographic area } \\
\hline Northeast & $147(30.3)$ & $144(29.7)$ & $291(30.0)$ \\
\hline Midwest & $201(41.4)$ & $206(42.5)$ & $407(42.0)$ \\
\hline South & $78(16.1)$ & $75(15.5)$ & $153(15.8)$ \\
\hline West & $59(12.2)$ & $60(12.4)$ & $119(12.3)$ \\
\hline \multicolumn{4}{|l|}{ Drug treatment } \\
\hline Levodopa MPR >0.8 & $321(66.2)$ & $298(61.4)$ & $619(63.8)$ \\
\hline DA $\mathrm{MPR}>0.8$ & $51(10.5)$ & $63(13.0)$ & $114(11.8)$ \\
\hline Both MPR >0.8 & $113(23.3)$ & $124(25.6)$ & $237(24.4)$ \\
\hline \multicolumn{4}{|l|}{ Receiving medication for } \\
\hline Pain & $269(55.5)$ & $331(68.2)$ & $600(61.9)$ \\
\hline Sleep disorders & $80(16.5)$ & $88(18.1)$ & $168(17.3)$ \\
\hline Depression & 237 (48.9) & $264(54.4)$ & $501(51.6)$ \\
\hline
\end{tabular}


A total of 485 patients were identified from the claims database as fulfilling all the study inclusion criteria for the "GIDs" group. Of these patients, $61 \%$ were adherent to levodopa only, $13 \%$ were adherent to DA only and $26 \%$ were adherent to both levodopa and DA regimes. The most common GIDs were oesophageal disorders (such as gastroesophageal reflux), digestive disorders (such as constipation) and dysphagia. A total of 485 matched controls were selected from patients who had PD but were not diagnosed with GIDs during the pre-index period. These matched controls formed the "No GIDs" group. Patients from the case and control groups exhibited similar demographic and clinical characteristics, such as age distribution, treatment regime and concurrent medications
(Table 4). Patients from the two groups had similar prescribed daily doses of levodopa and/or DA (data not shown).

Common symptoms of PD include sleep disorders, as well as dysfunctions of the neuropsychiatric, autonomic and sensory systems [4]. The incidence of these symptoms was examined via a search for associated ICD-9 diagnoses during the post-index period for both groups (Tables 3 and 4). The incidence of depression $(p=0.0347)$, anxiety $(p=0.0062)$, psychosexual dysfunction $(p=0.0499)$, ataxia $(p=0.0286)$, pain $(p=0.0003)$, movement disorders $(p=0.0053)$, urinary incontinence $(p=0.0156)$ and falls $(p=0.0356)$ was higher in the "GIDs" group compared with the "No GIDs" group (Tables 5 and 6, Figs 3 and 4).

Table 5

Incidence of selected neuropsychiatric diagnoses during the two year follow-up period of compliant PD patients with GIDs matched to compliant PD patients without GIDs

\begin{tabular}{lcccc}
\hline ICD-9 diagnosis & No GI disorder & GI disorder & Ratio & $p$ value \\
& $N=485$ & $N=485$ & GI : No GI & \\
\hline Dementia & $N(\%)$ & $N(\%)$ & & \\
Depression & $72(14.8)$ & $68(14.0)$ & 0.94 & 0.7129 \\
Hallucinations & $103(21.2)$ & $132(27.2)$ & 1.28 & 0.0347 \\
Delusions & $18(3.7)$ & $32(6.6)$ & 1.78 & 0.0508 \\
Anxiety & $2(0.4)$ & $3(0.6)$ & 1.50 & 0.6569 \\
Cognitive impairment & $46(9.5)$ & $74(15.3)$ & 1.61 & 0.0062 \\
Psychosexual dysfunction & $8(1.6)$ & $8(1.6)$ & 1.00 & 1.0000 \\
Hypersomnia & $1(0.2)$ & $8(1.6)$ & 8.00 & 0.0499 \\
Insomnia & $8(1.6)$ & $13(2.7)$ & 1.63 & 0.2800 \\
Aggression & $57(11.8)$ & $67(13.8)$ & 1.18 & 0.3366 \\
Ataxia & $0(0.0)$ & $3(0.6)$ & - & 0.9838 \\
Pain & $136(28.0)$ & $168(34.6)$ & 1.24 & 0.0286 \\
Autonomic neuropathy & $203(41.9)$ & $261(53.8)$ & 1.29 & 0.0003 \\
Mania & $1(0.2)$ & $2(0.4)$ & 2.00 & 0.5715 \\
Gambling & $0(0.0)$ & $4(0.8)$ & - & 0.9813 \\
Pathologic sexuality & $0(0.0)$ & $0(0.0)$ & - & N/A* \\
Aphasia & $0(0.0)$ & $0(0.0)$ & - & N/A* \\
Any of the above & $6(1.2)$ & $10(2.1)$ & 1.67 & 0.3226 \\
\hline
\end{tabular}

* $p$ values could not be calculated as no events occurred in either group.

Table 6

Incidence of selected motor, urogenital and cardiovascular disturbances as well as reported falls and fractures during the two year follow-up period of compliant PD patients with GIDs matched to compliant PD patients without GIDs

\begin{tabular}{|c|c|c|c|c|}
\hline ICD-9 diagnosis & $\begin{array}{c}\text { No GI disorder } \\
\begin{array}{c}N=485 \\
N(\%)\end{array}\end{array}$ & $\begin{array}{l}\text { GI disorder } \\
\begin{array}{c}N=485 \\
N(\%)\end{array}\end{array}$ & $\begin{array}{c}\text { Ratio } \\
\text { GI : No GI }\end{array}$ & $p$ value \\
\hline Motor disturbances: Movement disorder & $90(18.6)$ & $125(25.8)$ & 1.39 & 0.0053 \\
\hline Motor disturbances: Motor disorder & $0(0.0)$ & $0(0.0)$ & - & N/A* \\
\hline Motor disturbances: Coordination disorder & $36(7.4)$ & $50(10.3)$ & 1.39 & 0.1103 \\
\hline Motor disturbances: Any of the above & $115(23.7)$ & $158(32.6)$ & 1.37 & 0.0017 \\
\hline Urogenital disturbances: Urinary disorders & $65(13.4)$ & $66(13.6)$ & 1.02 & 0.9237 \\
\hline Urogenital disturbances: Urinary incontinence & $65(13.4)$ & $93(19.2)$ & 1.43 & 0.0156 \\
\hline Urogenital disturbances: Nocturia & $37(7.6)$ & $40(8.2)$ & 1.08 & 0.7221 \\
\hline Urogenital disturbances: Any of the above & $118(24.3)$ & $136(28.0)$ & 1.15 & 0.1852 \\
\hline Cardiovascular disturbances: Orthostatic hypotension & $31(6.4)$ & $43(8.9)$ & 1.39 & 0.1419 \\
\hline Falls and fractures: Falls & $50(10.3)$ & $72(14.8)$ & 1.44 & 0.0356 \\
\hline Falls and fractures: Fractures & $76(15.7)$ & $89(18.4)$ & 1.17 & 0.2674 \\
\hline Falls and fractures: Any of the above & $97(20.0)$ & $118(24.3)$ & 1.22 & 0.0989 \\
\hline
\end{tabular}

* $p$ values could not be calculated as no events occurred in either group. 


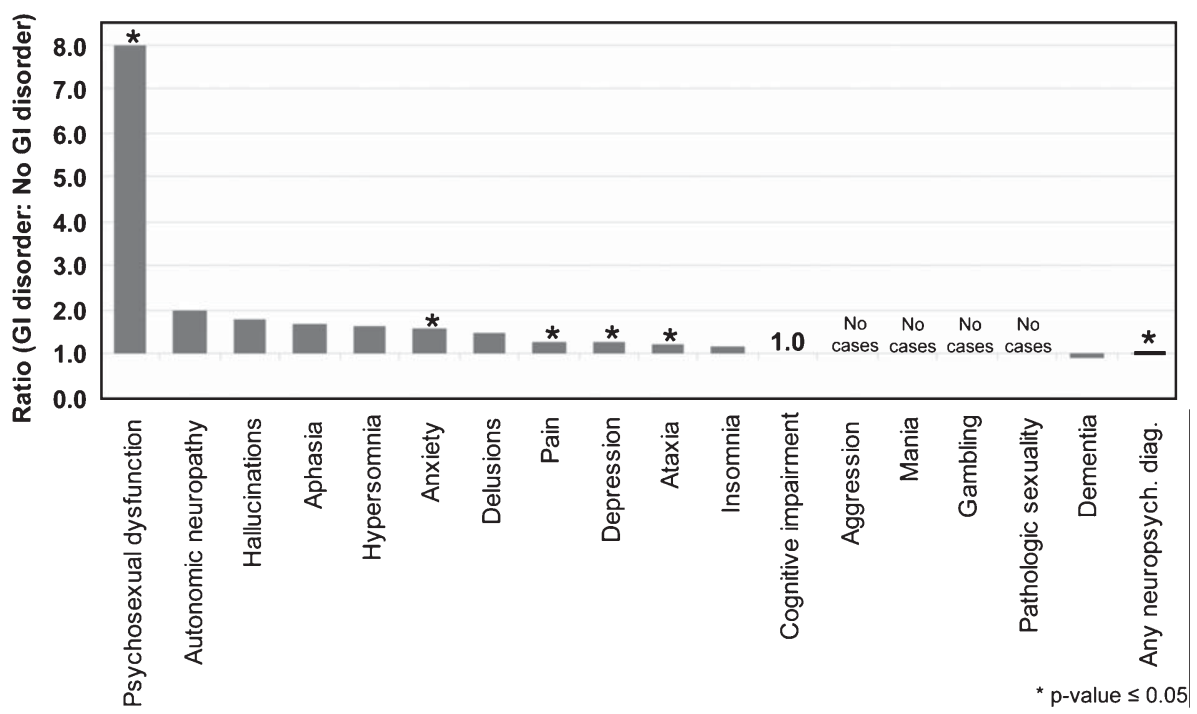

Fig. 3. Risk ratios for neuropsychiatric diagnoses during the two year follow-up period of compliant PD patients with GIDs matched to compliant PD patients without GIDs.

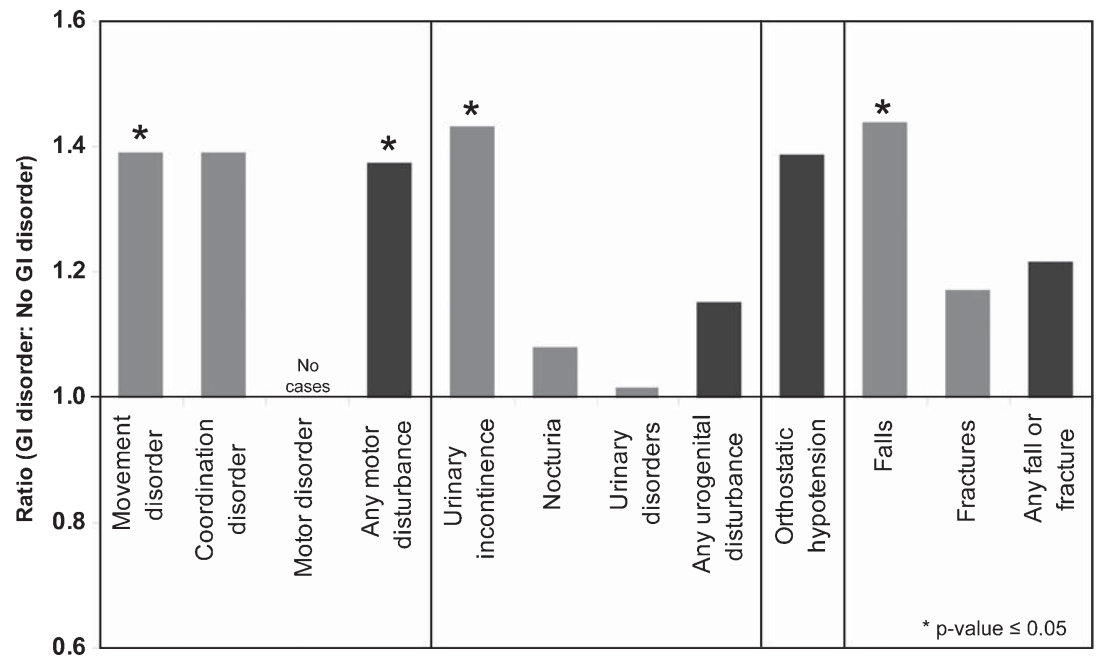

Fig. 4. Risk ratios for other PD-related comorbidities during the two year follow-up period of compliant PD patients with GIDs matched to compliant PD patients without GIDs.

Table 7

Mean values of selected healthcare utilization and economic outcomes during the two year follow-up period of compliant PD patients with GIDs matched to compliant PD patients without GIDs. These values are for the two years period, and thus are not annualised

\begin{tabular}{lrrcr}
\hline Outcome over two years & No GI disorder & GI disorder & $\begin{array}{c}\text { Ratio GI : No GI } \\
\text { GI : No GI }\end{array}$ & $p$ value \\
\hline Number ER visits & 1.245 & 1.771 & 1.42 & 0.0011 \\
Number neurologist visits & 20.973 & 20.660 & 0.99 & 0.8589 \\
Number hospitalizations & 0.744 & 0.819 & 1.10 & 0.6565 \\
Length of hospital stay & 7.553 & 6.825 & 0.90 & 0.9001 \\
Number drugs for pain, sleep, depression & 1.979 & 2.093 & 1.06 & 0.0432 \\
PD-related healthcare costs & $\$ 8,329$ & $\$ 9,384$ & 1.13 & 0.0038 \\
Non-PD-related healthcare costs & $\$ 27,780$ & $\$ 31,105$ & 1.12 & 0.0088 \\
Charlson Comorbidity Index & 1.975 & 2.113 & 1.07 & 0.2433 \\
\hline
\end{tabular}




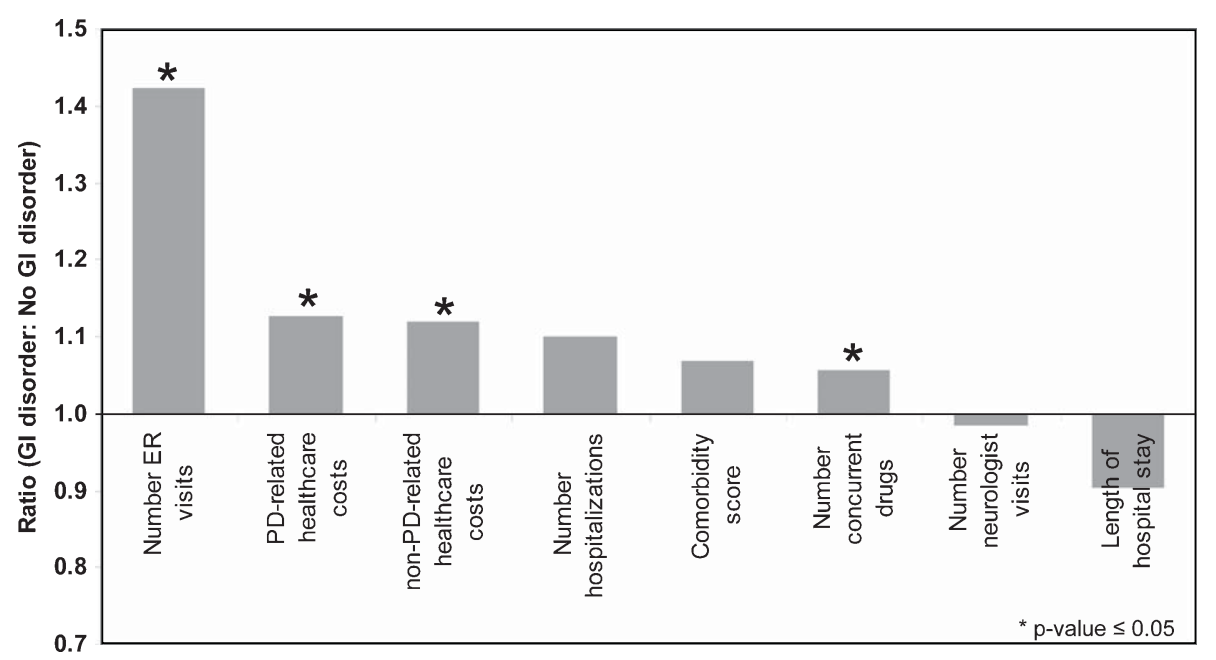

Fig. 5. Ratio of the mean healthcare and economic outcomes during the two year follow-up period of compliant PD patients with GIDs matched to compliant PD patients without GIDs.

Healthcare-related events and outcomes were examined during the post-index period (Table 7 and Fig. 5). The number of ER visits was found to be 1.42-fold higher in the "GIDs" group $(p=0.0011)$. The number of drugs prescribed for pain, sleep disorders, or depression was 1.06-fold higher in the "GIDs" group $(p=0.0432)$. Healthcare costs were also examined, and it was found that PD-related costs were 1.13-fold higher $(p=0.0038)$ while non-PD-related costs were 1.12 -fold higher $(p=0.0088)$ for the "GIDs" group than the "No GIDs group".

\section{DISCUSSION}

A retrospective claims database analysis was conducted to examine the longitudinal frequency of GIDs in PD, as well as the relationship between GIDs and PD-related outcomes. This longitudinal dataset suggests that $65 \%$ of compliant PD patients suffer from some form of GID four years after PD diagnosis. This is consistent with cross sectional and other observational data of patients with PD, as surveys have found that at least $66 \%$ of patients report at least one kind of GID, compared to about $29 \%$ of control patients [15].

Previous studies of claims databases found that patients with PD utilised more physicians, had higher physician-related costs and higher mortality than matched controls without PD [16, 17]. This is the first study to the authors' knowledge that examined the association between GIDs and PD-related outcomes using a retrospective claims database. This dataset demonstrates that patients with pre-existing GIDs before PD diagnosis have higher rates of some neurological, movement and urinary disorders. These patients also have higher rates of falls and ER visits in the first two years after their PD diagnosis. They have more prescriptions for drugs to treat pain, depression and sleep disorders than PD patients without GIDs.

Patients with GIDs had higher healthcare costs for both PD-related costs as well as those not directly related to PD. The total annual healthcare costs for compliant PD patients were found to be US\$18,055 for those without GIDs and US\$20,245 for those with GIDs. This is consistent with a previous finding of a total economic burden of $\$ 18,528$ for PD patients in the Medicare beneficiaries register [18].

This study has limitations that are intrinsic to the experimental design. While retrospective studies have the advantage of reflecting the healthcare utilisation of a large number of patients in an observational setting, these studies lack the level of control that exists in randomised studies. Strict causality cannot be demonstrated in an observational study, however, these studies are often essential in the generation of new hypotheses. Furthermore, while propensityscore matching was used to minimise the differences between the two groups, it is possible that these results may have been influenced by selection bias. Pre-index characteristics such as age, sex, US census region, plan type, comorbidities and treatment regime were included as covariates to ensure that the matched pairs were comparable. The administrative claims database utilized did not contain any supplemental patient data such as disease severity, tobacco use, or exposure to environmental toxins, and thus these variables were not able to be analysed or controlled for in this study. 
This study focused on compliant patients to ensure that medication non-adherence did not influence the results, and thus non-compliant PD patients may show a different association between GIDs and subsequent health outcomes.

These results add to the increasing body of evidence suggesting an association between GIDs and poorer PD-related outcomes. Increased retention within the stomach may convert some medications into a form that prevents intestinal absorption, and any delayed arrival of orally-delivered medication could cause erratic responses [5]. Gastric emptying influences the variation of the plasma bioavailability of treatment drugs, which may affect the response in patients with PD [19]. Indeed, delayed gastric emptying has been found to be more common in patients with PD who have "delayed-on" and "no-on" response fluctuations [20].

\section{CONCLUSION}

The majority of people diagnosed with PD will ultimately acquire at least one GID. This analysis used a US claims database to monitor GIDs incidence and found that it reached $65 \%$ at four years post PD diagnosis. To further examine the impact of GIDs on PD, a subset of compliant PD patients with GIDs was examined. These patients were matched with PD patients without GIDs and followed up for two years. Patients with GIDs had higher rates of psychosexual dysfunction, anxiety, depression, ataxia, pain, movement disorders, urinary incontinence and falls. Furthermore, ER admissions, number of comorbidity-related therapeutics and healthcare costs increased during the observation period in GIDs patients.

Overall, patients with both GIDs and PD appear to have worse health outcomes compared to patients who receive a diagnosis for $\mathrm{PD}$ with no prior GIDs diagnosis. Physicians treating PD patients should be aware that those patients with GIDs may be more likely to develop neurological, movement and urinary disorders and may require a higher level of care.

\section{ACKNOWLEDGEMENTS}

Declaration of interest: This study was funded by UCB Pharma S.A.

L.E.M., A.G. and F.R. are employees of UCB Pharma S.A., which manufactures various therapies including rotigotine for the treatment of PD. C.G. is a statistical programmer who was contracted by UCB
Pharma S.A. to assist in the analysis of epidemiological databases. The authors thank Soutrik Banerjee (Business \& Decision Life Sciences), Tatiana Kharkevitch (UCB Pharma S.A.), Frank Grieger (Schwarz Biosciences $\mathrm{GmbH}$, a member of the UCB Group), Chris Clark (UCB Inc.), Mike Davis (UCB Inc.) and Babak Boroojerdi (Schwarz Biosciences $\mathrm{GmbH}$ ) for interest in the work and discussion of the results, Ging-Ging Li (UCB Pharma S.A.) for publication coordination, Knut Mueller (Schwarz Biosciences $\mathrm{GmbH}$ ) for checking the quality of the SAS code and Carmela Semonelli (IMS Health) for assistance with the PharMetrics database.

\section{REFERENCES}

[1] Parkinson J (2002) An essay on the shaking palsy (Reprinted). Journal of Neuropsychiatry and Clinical Neurosciences, 14, 223-236.

[2] Rao SS, Hofmann LA, \& Shakil A (2006) Parkinson's disease: diagnosis and treatment. American Family Physician, 74, 2046-2056.

[3] Guttman M, Slaughter PM, Theriault ME, Dedoer DP, \& Naylor CD (2003) Burden of parkinsonism: a populationbased study. Movement Disorders, 18, 313-319.

[4] Chaudhuri KR, Healy DG, \& Schapira AHV (2006) Nonmotor symptoms of Parkinson's disease: diagnosis and management. Lancet Neurology, 5, 235-245.

[5] Pfeiffer RF (2003) Gastrointestinal dysfunction in Parkinson's disease. Lancet Neurology, 2, 107-116.

[6] Kaye J, Gage H, Kimber A, Storey L, \& Trend P (2006) Excess burden of constipation in Parkinson's disease: a pilot study. Movement Disorders, 21, 1270-1273.

[7] Verbaan D, Marinus J, Visser M, van Rooden SM, Stiggelbout AM, \& van Hilten JJ (2007) Patient-reported autonomic symptoms in Parkinson disease. Neurology, 69, 333-341.

[8] Antonini A, Tolosa E, Mizuno Y, Yamamoto M, \& Poewe WH (2009) A reassessment of risks and benefits of dopamine agonists in Parkinson's disease. Lancet Neurology, 8, 929937.

[9] Goole J \& Amighi K (2009) Levodopa delivery systems for the treatment of Parkinson's disease: an overview. International Journal of Pharmaceutics, 380, 1-15.

[10] Melamed E, Zoldan J, Galili-Mosberg R, Ziv I, \& Djaldetti R (1999) Current management of motor fluctuations in patients with advanced Parkinson's disease treated chronically with levodopa. Journal of Neural Transmission-Supplement, 173183.

[11] Brixner D (2006) Assessment of the prevalence and costs of osteoporosis treatment options in a real-world setting. American Journal of Managed Care, 12, S191-S198.

[12] Lau HS, de Boer A, Beuning KS, \& Porsius A (1997) Validation of pharmacy records in drug exposure assessment. Journal of Clinical Epidemiology, 50, 619-625.

[13] Deyo RA, Cherkin DC, \& Ciol MA (1992) Adapting a clinical comorbidity index for use with ICD-9-CM administrative databases. Journal of Clinical Epidemiology, 45, 613-619.

[14] Parsons LS (2001) Reducing bias in a propensity-score matched-pair sample using greedy matching techniques. Proceedings of the 26 th annual SAS ${ }^{\circledR}$ users group international conference, 214-226. 
[15] Edwards LL, Pfeiffer RF, Quigley EMM, Hofman R, \& Balluff M (1991) Gastrointestinal symptoms in Parkinson's disease. Movement Disorders, 6, 151-156.

[16] Guttman M, Slaughter PM, Theriault ME, Deboer DP, \& Naylor CD (2001) Parkinsonism in ontario-increased mortality compared with controls in a large cohort study. Neurology, 57, 2278-2282.

[17] Guttman M, Slaughter PM, Theriault ME, Deboer DP, \& Naylor CD (2002) Parkinsonism in ontario: physician utilization. Canadian Journal of Neurological Sciences, 29, 221-226.
[18] Noyes K, Liu HS, Li Y, Holloway R, \& Dick AW (2006) Economic burden associated with Parkinson's disease on elderly Medicare beneficiaries. Movement Disorders, 21 362-372.

[19] Muller T, Erdmann C, Bremen D, Schmidt WE, Muhlack S, Woitalla D, \& Goetze O (2006) Impact of gastric emptying on levodopa pharmacokinetics in Parkinson disease patients. Clinical Neuropharmacology, 29, 61-67.

[20] Djaldetti R, Baron J, Ziv I, \& Melamed E (1996) Gastric emptying in Parkinson's disease: patients with and without response fluctuations. Neurology, 46, 1051-1054. 